\title{
Ultrahigh Sensitivity Heavy Noble Gas Detectors for Long-Term Monitoring and for Monitoring Air DOE Award No. DE-FG07-99ER62758
}

\author{
Annual Report \\ $1 / 25 / 00$ \\ PI: Dr. John D. Valentine \\ Georgia Institute of Technology \\ Nuclear and Radiological Engineering Program \\ Atlanta, GA 30332-0405 \\ (404)894-3745 \\ John.Valentine@me.gatech.edu
}

\section{Research Objective}

The primary objective of this research project is to develop heavy noble gas (krypton, xenon, and radon) detectors for 1) long-term monitoring of transuranic waste, spent fuel, and other uranium and thorium bearing wastes and 2) alpha particle air monitors that discriminate between radon emissions and other alpha emitters. A University of Cincinnati/Argonne National Laboratory (UC/ANL) Team was assembled to complete this detector development project. Effective 1/4/99, the UC PI (John Valentine) became an Associate Professor in the Nuclear and Radiological Engineering Program of the George W. Woodruff School of Mechanical Engineering at the Georgia Institute of Technology. Consequently, this project was transferred to Georgia Tech (GT) with the PI. UC funding extended to 1/31/99 and GT funding became active 4/26/99. Since a previous Annual Report (submitted 7/2/99) summarized all of the achievements that were made at UC, this Annual Report will focus on work conducted at GT since 4/26/99 by the GT/ANL Team. DOE needs that are addressed by this project include improved long-term monitoring capability and improved air monitoring capability during remedial activities. Successful development and implementation of the proposed detection systems could significantly improve current capabilities with relatively simple and inexpensive equipment.

\section{Research Progress and Implications}

As of December 31, 1999, the GT/ANL Team has: 1) made significant progress toward characterizing the fluid transfer process which is the basis for this detector development project and 2) evaluated several radiation detectors and several potential pulse processing schemes.

ANL first developed the fluid transfer process through which heavy noble gases in the atmosphere are preferentially absorbed by certain organic fluids and can subsequently be degassed by adding a small amount of energy. It is this fluid transfer process that allows the heavy noble gases to be concentrated for enhanced detection. Due to ANL's extensive experience in characterizing this process, this part of the Team is continuing to take the lead in this area. Note, corn oil was used most extensively in this project at UC, however we have switched to 3-in-1 oil at GT due to its superior viscosity and because it does not polymerize with use as corn oil was observed to do. To further establish the characteristics of 3-in-1 oil for use in fluid transfer systems and to establish quantitative links between previous corn oil experiments and 3-in-1 oil experiments, a GT graduate student spent the Summer at ANL conducting pressure drop tests and sonication studies. Results indicated that 3-in-1 oil undergoes a smaller pressure drop than than corn oil when passing through one of the prototype absorption columns. Consequently, higher flow rates can be used and thus more air can be processed using 3-in-1 oil. This will undoubtedly be advantageous when scaling the prototype systems up to a fieldable system. As far as the sonication studies with 3-in-1 oil were concerned, the results were somewhat ambiguous. Nonetheless, results clearly indicated that sonication was an effective means of degassing 3-in-1 oil. However, further studies are required to determine the operating parameters that would be necessary to implement sonication in a fieldable system.

To complement these efforts, GT has constructed a dedicated walk-in hood facility for characterizing the fluid transfer processes, characterizing prototype concentration systems, and developing detection systems to be integrated with the fluid transfer concentration system. This facility 
is about 3 feet deep and 6 feet wide, with a height of about 7 feet. It should be noted that we were limited to system of about 20 inches in height at UC. Therefore, much larger fluid transfer prototypes can be characterized and used in this new facility. In addition, we have upgraded the controls of our prototype systems to be digital and primarily computer controllable. With these new controls, a custom LabView interface has been developed such that air and oil flow rates and other system parameters can be monitored and in many cases varied without human interaction during the experiment. Data acquisition software has also been integrated with a separate LabView interface such that a series of experiments (in which flow rates and data acquisition times and parameters can be varied, and different parts of the system can be turned on and off) can be conducted. This automation allows a much more efficient means of characterizing the fluid transfer processes, as well as detection systems. It also provides a more highly correlated set of data such that any spurious or otherwise unusual behavior can be more easily identified and its source determined.

To utilize this new facility, several new absorption columns and degassing units have been constructed and characterized. Results from these characterization studies are consistent with those of previous systems and associated scaling factors. In addition, these results are consistent with predictions made using simulations that were developed based on UC experiments. Both radon and Xe133 have been used in these studies to further establish how differences in solubility affect the concentration of these noble gases. With the larger dimensions of the new walk-in hood facility, prototype systems can be closer in scale to those of a fieldable system. Consequently, prototype results will not require as much extrapolation to estimate full-scale behavior.

To accurately detect and quantify some of the radioactive heavy noble gas isotopes, a detection scheme which distinguishes between alpha and beta particles, as well as recording gamma-ray spectra, results in the best system sensitivity. To accomplish this discrimination, potential detector candidates typically consist of one detector for the alpha and beta particles and a separate detector for the gamma rays. We have focussed on using a $\mathrm{NaI}(\mathrm{TI})$ scintillation detector as the gamma-ray detector due to its relatively good energy resolution and detection efficiency, and its amenability to field use. In addition, the GT/ANL Team has carefully evaluated a range of potential alpha/beta detectors and pulse processing techniques, including gas proportional detectors with pulse height discrimination, plastic scintillators with pulse shape discrimination, and silicon (PIPS) detectors with pulse height discrimination. The GT Team has recently evaluated a second generation of these detectors and pulse processing techniques. These systems typically consisted of two alpha/beta detectors and two gammaray detectos, and the pulse processing electronics necessary to combine and evaluate the signals from these detectors. Based on results from these experiments, it appears as if the PIPS/NaI(Tl) combination will provide the best sensitivity and should thus be used in any fieldable high-sensitivity system. However, it was also concluded that the gas proportional detector/ $\mathrm{NaI}(\mathrm{Tl})$ combination was typically sufficient for characterizing the fluid transfer process, and in many cases easier to implement.

An additional conclusion from these studies was that more sophisticated beta/gamma coincidence techniques need to be used to fully utilize these detector configurations. By only recording decays in which two or more emissions (typically a beta particle or conversion electron and a gamma ray) are detected in coincidence, background counts can be significantly reduced. Such coincidence pulse processing techniques were used with the gas proportional detector/NaI(Tl), plastic scintillator/NaI(T1), and PIPS/NaI(Tl). However, a pulse processing system that allows dual-parameter pulse height analysis of coincidence events provides even better sensitivity and a superior ability to distinguish the characteristic signatures of different isotopes. A dual-parameter data acquisition system has been procured and, as indicated in the Planned Activities Section below, will be used to further develop high-sensitivity detection.

To better understand the behavior of $\mathrm{NaI}(\mathrm{Tl})$ as the gamma-ray spectroscopy detector, we have studied the effect of light yield nonproportionality on scintillator energy resolution. These studies involve trying to decouple this effect from the total energy resolution and are being pursued both experimentally and computationally. It is possible that developing an understanding of these effects and techniques for characterizing them will provide guidance in developing new scintillators with performance characteristics superior to $\mathrm{NaI}(\mathrm{Tl})$.

One additional application that has been identified for use with the fluid transfer techniques is radon mitigation. By processing ambient air with a fluid transfer system, it may be possible to significantly reduce radon concentrations. Not only is this a significant issue in households, but such technology could significantly facilitate some DOE EM activities in areas where radon levels are high. Preliminary studies have been conducted by the GT Team to begin to establish the ability of the fluid 
transfer process to mitigate radon. While preliminary results indicate that radon concentration can be measurably reduced from small volumes, further studies are necessary to determine the full capabilities of fluid transfer in mitigating radon.

In summary, the GT/UC/ANL Team has made significant progress toward achieving the goals of this project. While fieldable systems have not been realized to date, all of the pieces are now in place to start going from lab-scale to field-scale systems primarily due to the achievements thus far. When such fieldable systems are available, DOE EM activities will be significantly enhanced in the air monitoring and long-term monitoring areas. There also exists a significant opportunity for this technology to be applied in radon mitigation.

\section{Planned Activities}

The GT/ANL Team will focus on two major areas over the next year. First, we will focus on further characterizing the absorption and degassing mechanisms associated with the fluid transfer concentration system. This effort will consist of conducting experiments with spiked radioactive xenon and radon samples and subsequently analyzing the results using radiation detection systems. In addition, more extensive and systematic series of experiments will be conducted to characterize the absorption and degassing mechanisms, as well as optimize the fluid transfer concentration system for fieldable implementation. The second major focus area for the next year consists of further development of application specific detector and pulse processing techniques. While we have developed significant expertise and knowledge in this area to date, the effort will become more focussed and application specific. We will also implement the dual parameter pulse height analysis system to further improve system sensitivity. We will also continue to study the effect of light yield nonproportionality on scintillator energy resolution. Finally, studies will be conducted to better characterize the capability of the fluid transfer process for radon mitigation. In the final year of the project, the GT/ANL Team will integrate these two efforts to develop a fieldable system (or systems) consisting of an optimized fluid transfer concentration system and an optimized detection system.

\section{Information Access}

The GT/ANL Team presented two papers at the $218^{\text {th }}$ ACS National Meeting describing the performance of a small prototype fluid transfer system and the solubility of heavy noble gases in trigliceride oil. In addition, we presented one paper at the 1999 IEEE Nuclear Science Symposium describing the performance of a second generation of detectors and pulse processing techniques. The latter paper has been submitted for publication in IEEE Transactions on Nuclear Science. 